\title{
Existence and Uniqueness of Load-Flow Solutions in Three-Phase Distribution Networks
}

\author{
Cong Wang, Student Member, IEEE, Andrey Bernstein, Member, IEEE, Jean-Yves Le Boudec, Fellow, IEEE, and \\ Mario Paolone, Senior Member, IEEE
}

\begin{abstract}
We present sufficient conditions for the existence and uniqueness of load-flow solutions in three-phase distribution networks. The conditions can be efficiently verified for real distribution systems.
\end{abstract}

Index Terms-load flow solution, fixed point method, existence and uniqueness, distribution networks.

\section{INTRODUCTION}

$\mathbf{I}$ $\mathrm{N}$ distribution networks, many control procedures rely on the existence and uniqueness of load-flow solutions. However, due to the non-linearity of the load-flow equations, these properties are difficult to study. In this letter, we give efficiently verifiable conditions that guarantee the existence and uniqueness of load-flow solutions in three-phase distribution networks.

\section{Problem Formulation}

We consider a three-phase network that has one slack bus indexed by $0, N P Q$ buses indexed by $1, \ldots, N$, and a generic topology (i.e., radial or meshed). Let $\mathbf{v}_{j} \triangleq\left(v_{j}^{a}, v_{j}^{b}, v_{j}^{c}\right)^{T}$, $\mathbf{s}_{j} \triangleq\left(s_{j}^{a}, s_{j}^{b}, s_{j}^{c}\right)^{T}$ be the complex vectors representing threephase nodal voltage and power injection at bus $j \in\{0, \ldots, N\}$, and define $\mathbf{v} \triangleq\left(\mathbf{v}_{1}^{T}, \ldots, \mathbf{v}_{N}^{T}\right)^{T}, \mathbf{s} \triangleq\left(\mathbf{s}_{1}^{T}, \ldots, \mathbf{s}_{N}^{T}\right)^{T}$. Complex conjugates are denoted by adding an overline ${ }^{-}$. Then, the loadflow problem consists in solving for $\mathbf{v}$ and $\mathbf{s}_{0}$ in the following equations, where $\mathbf{s}$ and $\mathbf{v}_{0}$ are given and $\mathbf{Y}$ is the three-phase compound admittance matrix [1]:

$$
\left[\begin{array}{c}
\mathbf{s}_{0} \\
\mathbf{s}
\end{array}\right]=\left[\begin{array}{ll}
\operatorname{diag}\left(\mathbf{v}_{0}\right) & \\
& \operatorname{diag}(\mathbf{v})
\end{array}\right] \overline{\mathbf{Y}}\left[\begin{array}{c}
\overline{\mathbf{v}}_{0} \\
\overline{\mathbf{v}}
\end{array}\right]
$$

Notice that matrix $\mathbf{Y}$ can be partitioned as

$$
\mathbf{Y}=\left[\begin{array}{cc}
\mathbf{Y}_{00} & \mathbf{Y}_{0 L} \\
\mathbf{Y}_{L 0} & \mathbf{Y}_{L L}
\end{array}\right]
$$

with $3 \times 3$ matrix $\mathbf{Y}_{00}, 3 \times 3 N$ matrix $\mathbf{Y}_{0 L}, 3 N \times 3$ matrix $\mathbf{Y}_{L 0}$, and $3 N \times 3 N$ matrix $\mathbf{Y}_{L L}$ (see Section IV-C for its invertibility). Then by defining the zero-load voltage $\mathbf{w} \triangleq-\mathbf{Y}_{L L}^{-1} \mathbf{Y}_{L 0} \mathbf{v}_{0}=\left(w_{1}^{a}, w_{1}^{b}, w_{1}^{c}, \ldots, w_{N}^{a}, w_{N}^{b}, w_{N}^{c}\right)^{T}$, the load-flow problem can be reduced to Eq.(1), where $\mathbf{v}$ is unknown and $\mathbf{s}$ is given :

$$
\mathbf{v}=\mathbf{w}+\mathbf{Y}_{L L}^{-1} \operatorname{diag}(\overline{\mathbf{v}})^{-1} \overline{\mathbf{s}}
$$

The work is supported by SNSF-NRP70 "Energy Turnaround". The authors are with École Polytechnique Fédérale de Lausanne (EPFL), CH-1015 Lausanne, Switzerland (e-mail: firstname.lastname@epfl.ch).
Eq.(1) is called the implicit $Z_{\text {bus }}$ formulation [2]; it is a fixed point equation in $\mathbf{v}$ and can be solved by the iterative scheme in Eq.(2) :

$$
\mathbf{v}^{(k+1)}=\mathbf{w}+\mathbf{Y}_{L L}^{-1} \operatorname{diag}\left(\overline{\mathbf{v}}^{(k)}\right)^{-1} \overline{\mathbf{s}}
$$

In the rest of this letter, we give sufficient conditions under which there exists a unique load-flow solution that can be found by the iteration in Eq.(2).

\section{RESULTS}

In Theorem 1 we give a result assuming that we have prior knowledge of one pair $(\hat{\mathbf{v}}, \hat{\mathbf{s}})$ that satisfies Eq.(1). This is true in the cases where the load-flow problem is repeatedly solved for varying operational conditions (namely, $\hat{\mathbf{v}}$ is a solution computed in a previous instance of the problem when the power injection was $\hat{\mathbf{s}})$, or where a pair $(\hat{\mathbf{v}}, \hat{\mathbf{s}})$ is obtained by other methods. In Corollary 1, we give a result that does not depend on such prior knowledge.

The result makes use of the following notation :

$$
\begin{gathered}
\xi(\mathbf{s}) \triangleq\left\|\mathbf{W}^{-1} \mathbf{Y}_{L L}^{-1} \overline{\mathbf{W}}^{-1} \operatorname{diag}(\overline{\mathbf{s}})\right\|_{\infty} \\
u_{\min }(\mathbf{v}) \triangleq \min _{j \in\{1, \ldots, N\}, \gamma \in\{a, b, c\}}\left|v_{j}^{\gamma} / w_{j}^{\gamma}\right| \\
\mathcal{D}(\rho, \hat{\mathbf{v}}) \triangleq\left\{\mathbf{v}:\left|v_{j}^{\gamma}-\hat{v}_{j}^{\gamma}\right| \leq \rho\left|w_{j}^{\gamma}\right|, j \in\{1, \ldots, N\}, \gamma \in\{a, b, c\}\right\} .
\end{gathered}
$$

In the above, $\mathbf{W} \triangleq \operatorname{diag}(\mathbf{w})$, and $\|\bullet\|_{\infty}$ is the matrix norm induced by the $\ell^{\infty}$ norm (i.e., $\|A\|_{\infty}=$ $\left.\max _{1 \leq j \leq 3 N} \sum_{k=1}^{3 N}\left|A_{j, k}\right|\right)$.

Theorem 1. Let $\hat{\mathbf{v}}$ be a solution to Eq.(1) with power injection $\hat{\mathbf{s}}$ and assume that $\left(u_{\min }(\hat{\mathbf{v}})\right)^{2}>\xi(\hat{\mathbf{s}})$.

For any other power injection vector $\mathbf{s}$ that satisfies

$$
\xi(\mathbf{s}-\hat{\mathbf{s}})<\frac{1}{4}\left(u_{\min }(\hat{\mathbf{v}})-\xi(\hat{\mathbf{s}}) / u_{\min }(\hat{\mathbf{v}})\right)^{2}
$$

there is a unique solution $\mathbf{v}$ in $\mathcal{D}\left(\rho^{\ddagger}, \hat{\mathbf{v}}\right)$ to Eq.(1) with power injection $\mathbf{s}$, where $\rho^{\ddagger} \triangleq \frac{1}{2}\left(u_{\min }(\hat{\mathbf{v}})-\xi(\hat{\mathbf{s}}) / u_{\min }(\hat{\mathbf{v}})\right)$.

This unique solution can be reached by applying the iteration in Eq.(2) initialized with any $\mathbf{v}^{(0)} \in \mathcal{D}\left(\rho^{\ddagger}, \hat{\mathbf{v}}\right)$.

Moreover, this unique solution is located in the smaller domain $\mathcal{D}\left(\rho^{\dagger}, \hat{\mathbf{v}}\right)$ with

$$
\rho^{\dagger} \triangleq \rho^{\ddagger}-\frac{1}{2} \sqrt{\left(u_{\min }(\hat{\mathbf{v}})-\xi(\hat{\mathbf{s}}) / u_{\min }(\hat{\mathbf{v}})\right)^{2}-4 \xi(\mathbf{s}-\hat{\mathbf{s}})} .
$$

Observe that the localization in the smaller domain $\mathcal{D}\left(\rho^{\dagger}, \hat{\mathbf{v}}\right)$ is more accurate but depends (via $\rho^{\dagger}$ ) on the specific $\mathbf{s}$, unlike the larger domain $\mathcal{D}\left(\rho^{\ddagger}, \hat{\mathbf{v}}\right)$. Also note that the theorem implies that there is no solution $\mathbf{v}$ in $\mathcal{D}\left(\rho^{\ddagger}, \hat{\mathbf{v}}\right) \backslash \mathcal{D}\left(\rho^{\dagger}, \hat{\mathbf{v}}\right)$.

If no $(\hat{\mathbf{v}}, \hat{\mathbf{s}})$ is obtained, we can use the following corollary. 
Corollary 1. Suppose that the power injection $\mathbf{s}$ satisfies $\xi(\mathbf{s})<0.25$. There exists a unique solution $\mathbf{v}$ in $\mathcal{D}(0.5, \mathbf{w})$ to Eq.(1) with power injection $\mathbf{s}$. This solution can be reached by applying the iteration in Eq.(2) initialized with any $\mathbf{v}^{(0)} \in$ $\mathcal{D}(0.5, \mathbf{w})$. Moreover, it is located in the smaller domain $\mathcal{D}(\rho, \mathbf{w})$ with $\rho=(1-\sqrt{1-4 \xi(\mathbf{s})}) / 2$.

Note that all the proposed conditions on $\hat{\mathbf{v}}, \hat{\mathbf{s}}, \mathbf{s}$ can be verified at low computational complexity prior to solving a load-flow problem. Thus, they can be used for network control applications like Distribution Management Systems that need to solve multiple instances of load-flow problems in real time.

Remark 1. Theorem 1 and Corollary 1 are essentially extension to general three-phase distribution networks of the main results for single-phase networks in [3]. The key steps of this extension are (i) formulating the three-phase loadflow problem in the same algebraic form as its single-phase counterpart, and (ii) proving the invertibility of matrix $\mathbf{Y}_{L L}$ in three-phase networks.

\section{PROOFS}

\section{A. Proof of Theorem 1}

Proof. The three-phase implicit $Z_{b u s}$ formulation in Eq.(1) has exactly the same algebraic form as its single-phase counterpart in [3], thus we can directly follow and apply the proof of Lemma 1 and Lemma 2 in [3]. Let $\mathbf{G}(\mathbf{v})$ express the righthandside of Eq.(1). It follows that, whenever the conditions in Theorem 1 are true, $\mathbf{G}()$ is a self-mapping and contraction mapping on $\mathcal{D}(\rho, \hat{\mathbf{v}})$ for any $\rho \in\left[\rho^{\dagger}, \rho^{\ddagger}\right]$. Therefore, according to Banach's fixed point theorem [4], there is a unique solution in $\mathcal{D}\left(\rho^{\dagger}, \hat{\mathbf{v}}\right)$ to Eq.(1), and it can be reached by the iteration in Eq.(2) for any $\mathbf{v}^{(0)} \in \mathcal{D}\left(\rho^{\dagger}, \hat{\mathbf{v}}\right)$; the same also holds if we replace $\rho^{\dagger}$ by $\rho^{\ddagger}$. Since $\mathcal{D}\left(\rho^{\dagger}, \hat{\mathbf{v}}\right) \subseteq \mathcal{D}\left(\rho^{\ddagger}, \hat{\mathbf{v}}\right)$, we conclude that (i) there is a unique solution in $\mathcal{D}\left(\rho^{\ddagger}, \hat{\mathbf{v}}\right)$; (ii) the solution is located in $\mathcal{D}\left(\rho^{\dagger}, \hat{\mathbf{v}}\right)$; (iii) it can be reached by iteration in Eq.(2) with any $\mathbf{v}^{(0)} \in \mathcal{D}\left(\rho^{\ddagger}, \hat{\mathbf{v}}\right)$.

\section{B. Proof of Corollary 1}

Proof. Apply Theorem 1 with $\hat{\mathbf{v}}=\mathbf{w}$ and $\hat{\mathbf{s}}=\mathbf{0}$.

\section{Invertibility of $\mathbf{Y}_{L L}$}

The admittance matrix depends on specific device modeling. Here, we show that $\mathbf{Y}_{L L}$ is invertible when the following system assumptions hold, which covers most practical cases.

- The longitudinal component and shunt elements of transmission line between any pair of buses are described by circulant matrices;

- Transformer between any pair of buses is depicted by models in [5], but equipped with complex ratio;

- The connection from the slack bus to a $P Q$ bus can be realized via (i) a transmission line, or (ii) a transformer of either Delta $-\mathrm{Wye}_{\mathrm{G}}$ or $\mathrm{Wye}_{\mathrm{G}}-\mathrm{Wye}_{\mathrm{G}}$ configuration;

- The connection between two $P Q$ buses can be established through either a transmission line or a transformer of $\mathrm{Wye}_{\mathrm{G}}-\mathrm{Wye}_{\mathrm{G}}$ configuration;

- Transformers may have additional core losses in the form of transverse components attached to related buses;
- Transmission lines and transformers do not generate active power, and their longitudinal components have positive resistance in zero-, positive-, negative sequences.

Proof. To prove that $\mathbf{Y}_{L L}$ is invertible we show that if $\mathbf{x} \in$ $\mathbb{C}^{3 N}$ is a vector such that $\mathbf{Y}_{L L} \mathbf{x}=0$ then we must have $\mathbf{x}=\mathbf{0}$. We can view $\mathbf{Y}_{L L}$ as the admittance matrix of a fictitious $N$-bus network (i.e., the original network with the slack bus grounded) and $\mathbf{x}$ as its nodal voltage vector; then the sum of all nodal power injections of this $N$-bus network is $s^{\text {total }}=\mathbf{x}^{T} \overline{\mathbf{Y}}_{L L} \overline{\mathbf{x}}=0$.

Let $\mathcal{V}^{\text {slack }}$ be the set of buses that are connected with the slack bus in the original $N+1$-bus network, and write $\mathbf{x}$ as $\mathbf{x}=\left(\mathbf{x}_{1}^{T}, \ldots, \mathbf{x}_{N}^{T}\right)^{T}$ with $\mathbf{x}_{j}, j \in\{1, \ldots, N\}$ interpreted as the three-phase nodal voltage at bus $j$. Note that $s^{\text {total }}$ equals the system power loss and can be further decomposed as

$$
s^{\text {total }}=s^{\text {slack }}+s^{\text {line }}+s^{\text {shunt }}+s^{\text {leakage }}+s^{\text {core-loss }}
$$

where

- $s^{\text {slack }}$ is the power consumption in the transverse components that result from connections to the slack bus in the original $N+1$-bus network of all buses $j \in \mathcal{V}^{\text {slack; }}$

- $s^{\text {line }}$ is the power consumption in the longitudinal components of all transmission lines;

- $s^{\text {shunt }}$ is the power consumption in all shunt elements;

- $s^{\text {leakage }}$ is the power consumption that results from all transformer leakage impedances;

- $s^{\text {core-loss }}$ is the power consumption caused by core losses in all transformers.

By the last item of assumption, $s^{\text {total }}=0$ implies that all the five terms contain zero real parts. Therefore, according to the system assumptions, we have

1) $\Re\left(s^{\text {slack }}\right)=0$ implies that $\mathbf{x}_{j}=\mathbf{0}$ for all $j \in \mathcal{V}^{\text {slack; }}$

2) From $\Re\left(s^{\text {line }}\right)=0$, it can be obtained that $\mathbf{x}_{j}=\mathbf{x}_{l}$ for transmission line between any buses $j, l \in\{1, \ldots, N\}$;

3) By $\Re\left(s^{\text {leakage }}\right)=0$ and the $\mathrm{Wye}_{\mathrm{G}}-\mathrm{Wye}_{\mathrm{G}}$ configuration, we have $\mathbf{x}_{j}=K_{j l} \mathbf{x}_{l}$ for transformer with ratio $K_{j l}$ between any buses $j, l \in\{1, \ldots, N\}$.

From the first item, there is at least one bus that has zero voltage. From the second and the third items, the zero voltage propagates throughout the $N$-bus network. Thus, we have $\mathbf{x}=$ 0.

\section{REFERENCES}

[1] J. Arrillaga, D. Bradley, and P. Bodger, Power System Harmonics. Chicester, U.K.: Wiley, 1985.

[2] T.-H. Chen, M.-S. Chen, K.-J. Hwang, P. Kotas, and E. A. Chebli, "Distribution system power flow analysis - a rigid approach," IEEE Trans. on Power Deliv., vol. 6, no. 3, pp. 1146-1152, Jul. 1991.

[3] C. Wang, A. Bernstein, J.-Y. Le Boudec, and M. Paolone, "Explicit conditions on existence and uniqueness of load-flow solutions in distribution networks," IEEE Transactions on Smart Grid, 2016, DOI 10.1109/TSG.2016.2572060.

[4] A. Quarteroni, R. Sacco, and F. Saleri, Numerical mathematics, 2nd ed. Berlin, Germany: Springer, 2007.

[5] M. S. Chen and W. E. Dillon, "Power system modeling," Proceedings of the IEEE, vol. 62, no. 7, pp. 901-915, Jul. 1974. 